\title{
Correction to: Robotic Surgery in Pediatric Oncology: Lessons Learned from the First 100 Tumors-A Nationwide Experience
}

\author{
Thomas Blanc, MD, PhD ${ }^{1,2,3}$ (D) , Pierre Meignan, $\mathbf{M D}^{1,4}$, Nicolas Vinit, $\mathrm{MD}^{1}$, Quentin Ballouhey, PhD ${ }^{5}$, \\ Luca Pio, MD ${ }^{1}$, Carmen Capito, $\mathrm{PhD}^{1}$, Caroline Harte, $\mathrm{MD}^{6}$, Fabrizio Vatta, $\mathrm{MD}^{1}$, Louise Galmiche-Rolland, $\mathrm{PhD}^{7}$, \\ Véronique Minard, $\mathbf{P h D}^{8}$, Daniel Orbach, $\mathbf{P h D}^{9}$, Laureline Berteloot, $\mathbf{M D}^{10}$, Cécile Muller, $\mathbf{P h D}^{1}$, \\ Jules Kohaut, MD ${ }^{1}$, Aline Broch, $\mathrm{MD}^{1}$, Karim Braik, $\mathrm{MD}^{4}$, Aurélien Binet, $\mathrm{MD}^{4}$, Yves Heloury, PhD ${ }^{1,2}$, \\ Laurent Fourcade, $\mathrm{PhD}^{5}$, Hubert Lardy, $\mathrm{PhD}^{4}$, and Sabine Sarnacki, $\mathbf{P h D}^{1,2}$ \\ ${ }^{1}$ Department of Pediatric Surgery and Urology, Hôpital Necker-Enfants Malades, Assistance Publique-Hôpitaux de Paris, \\ Paris, France; ${ }^{2}$ Université de Paris, Paris, France; ${ }^{3}$ Département « Croissance et Signalisation », Centre National de la \\ Recherche Scientifique UMR8253, Institut National de la Santé et de la Recherche Médicale U1151, Institut Necker \\ Enfants Malades, Université de Paris, Paris, France; ${ }^{4}$ Department of Pediatric Surgery, Hôpital Clocheville, Tours, France; \\ ${ }^{5}$ Department of Pediatric Surgery, Hôpital des Enfants, Limoges, France; ${ }^{6}$ Department of Pediatric Anesthesia, Hôpital \\ Necker-Enfants Malades, Assistance Publique-Hôpitaux de Paris, Paris, France; ${ }^{7}$ Department of Pathology, Hôpital \\ Necker-Enfants Malades, Assistance Publique-Hôpitaux de Paris, Paris, France; ${ }^{8}$ Department of Pediatric and Adolescent \\ Oncology, Institut Gustave Roussy, Villejuif, France; ${ }^{9}$ Department of Pediatric Oncology SIREDO Oncology Center (Care, \\ Innovation and Research for Children and AYA with Cancer), Institut Curie, PSL Research University, Paris, France; \\ ${ }^{10}$ Department of Pediatric Radiology, Hôpital Necker-Enfants Malades, Assistance Publique-Hôpitaux de Paris, Paris, \\ France
}

\section{CORRECTION TO: ANN SURG ONCOL HTTPS://DOI.ORG/10.1245/S10434-021-10777-6}

Luca Pio's given name is correct as reflected here. The original article was corrected.
Publisher's Note Springer Nature remains neutral with regard to jurisdictional claims in published maps and institutional affiliations.

The original article can be found online at https://doi.org/10.1245/ s10434-021-10777-6.

(C) Society of Surgical Oncology 2021

Published Online: 2 October 2021

T. Blanc, $\mathrm{MD}, \mathrm{PhD}$

e-mail: thomas.blanc@aphp.fr 